\title{
Narcissism in patients admitted to psychiatric acute wards: its relationship to violence, suicidality and other psychopathology Marit Svindseth* and Alv A Dahl
}

Address: Department of Clinical Cancer Research, Rikshospitalet/Radiumhospitalet, 0310 Oslo, Norway

* Corresponding author

from WPA Thematic Conference. Coercive Treatment in Psychiatry: A Comprehensive Review

Dresden, Germany. 6-8 June 2007

Published: 19 December 2007

BMC Psychiatry 2007, 7(SuppI I):SII5 doi:10.1 I86/I47I-244X-7-SI-SII5

This abstract is available from: http://www.biomedcentral.com/I47I-244X/7/SI/SII5

(c) 2007 Svindseth and Dahl; licensee BioMed Central Ltd.

\section{Background}

Although associations between psychopathology, violence, and suicidality and narcissism have been demonstrated, these associations have hardly been studied in patients admitted to acute psychiatric wards. The objective was to examine these associations in such a sample.

\section{Methods}

Eligible patients were interviewed with the Brief Psychiatric Rating Scale (BPRS) and filled in the Narcissistic Personality Inventory (NPI). Violence, Global Assessment of Functioning scores (GAF), and ICD-10 diagnoses were collected from medical records.

\section{Results}

Among 351 patients, 186 were eligible and gave informed consent (98 involuntary and 88 voluntary admitted). In correlation analyses of the BPRS and NPI ratings, depression and suicidality were negatively correlated with the narcissism scores. Severe violence, grandiosity and hyperactivity were positively correlated with narcissism. The sample was separated at the median total score of the NPI into subsamples with high and low narcissism. A significant negative association was observed between both GAF and self-esteem scores and narcissism. The group with high narcissism was significantly younger, contained more males, more involuntary admissions, more severe violence, more frequently a diagnosis of schizophrenia, less frequently suicidal. Multivariable logistic regression analyses showed that severe violence was positively associated with high narcissism, while low self-esteem, less suicidality and lower age was negatively associated with high narcissism.

\section{Conclusion}

In patients admitted to acute wards we found associations between violence, hyperactivity and involuntary admission and high narcissism. High narcissism was associated with reduced suicidality and level of depression. These findings can be useful in clinical settings and ratings of narcissism may be useful in prediction of violence. 